\title{
ChemComm
}

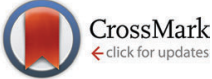

Cite this: Chem. Commun., 2016, 52, 13596

Received 20th September 2016, Accepted 19th October 2016

DOI: $10.1039 / c 6 c c 07632 g$

www.rsc.org/chemcomm

\section{Importance of the structural integrity of a carbon conjugated mediator for photocatalytic hydrogen generation from water over a CdS-carbon nanotube- $\mathrm{MoS}_{2}$ composite $\dagger$}

\author{
Molly Meng-Jung Li, ${ }^{a}$ Poppy Mills, ${ }^{a}$ Simon M. Fairclough, $\neq^{a}$ Alex Robertson, ${ }^{b}$ \\ Yung-Kang Peng, ${ }^{a}$ Jamie Warner, ${ }^{b}$ Chunyang Nie, ${ }^{c}$ Emmanuel Flahaut ${ }^{c}$ and \\ Shik Chi Edman Tsang*a
}

Incorporation of CdS quantum dots is shown to significantly promote photocatalytic hydrogen production from water over singlelayer $\mathrm{MoS}_{2}$ in a remote manner via their dispersions on a carbon nanotube as a nanocomposite: the hydrogen evolution rate is found to be critically dependent on the content and structural integrity of the carbon nanotube such that the double-walled carbon nanotube shows superior $\mathrm{H}_{2}$ production to a single-walled carbon nanotube because the inner carbon tubules survive from the structural damage during functionalization.

The depletion of fossil fuel reserves and the undeniable environmental harm caused by their overconsumption make photocatalytic $\mathrm{H}_{2}$ production from water attractive as a future renewable energy source. ${ }^{1,2}$ Typically, photocatalytic splitting of water requires semiconductors to capture a photon with energy equal to or greater than their band gap energy. The photons are absorbed by a semiconductor nanocrystal, causing the generation of photoexcited electrons and holes in the conduction and valence bands, respectively. The excited electron-hole pairs, known as excitons, are then subjected to two main competitive deactivation routes, namely exciton recombination and exciton separation followed by chemical reactions. The former route is undesirable in photocatalysis, since this only emits heat or light with no chemical process involved, whereas the latter is important for photocatalytic reduction of water to hydrogen gas.

Nanosize CdS is a quantum dot material for efficient capture and emission of photons due to its characteristic band edges and band gap. ${ }^{3,4}$ However, it displays very poor photocatalytic

\footnotetext{
${ }^{a}$ Wolfson Catalysis Centre, Department of Chemistry University of Oxford, Oxford, OX1 3QR, UK. E-mail: edman.tsang@chem.ox.ac.uk

${ }^{b}$ Department of Materials, University of Oxford, Oxford, OX1 3PH, UK ${ }^{c}$ Centre Interuniversitaire de Recherche et d'Ingénierie des Matériaux, Université Paul Sabatier, CIRIMAT, UMR CNRS 5085, Toulouse, France $\dagger$ Electronic supplementary information (ESI) available: Supporting figures, and detailed experimental procedures, including XRD, TEM, FTIR, Raman, etc. See DOI: $10.1039 / \mathrm{c} 6 \mathrm{cc} 07632 \mathrm{~g}$

\$ Present address: Department of Physics, King’s College London, UK.
}

activity due to rapid exciton recombination. ${ }^{5,6}$ On the other hand, molybdenum disulfide $\left(\mathrm{MoS}_{2}\right)$ is not an intrinsically active semiconductor to capture photons in photo-catalysis but its conduction band $(-0.12 \mathrm{eV} v s$. NHE) and valance band $(1.78 \mathrm{eV}$ vs. NHE) render it suitable for electrochemical water-splitting. ${ }^{7}$ It has been reported that an intimate $\mathrm{CdS} / \mathrm{MoS}_{2}$ mixture can catalyze photocatalytic $\mathrm{H}_{2}$ evolution efficiently. ${ }^{8}$ In addition, CdS supported on carbon materials is also proven to suppress charge recombination and provide active adsorption sites, which leads to the enhancement of photocatalytic activity. ${ }^{9-13}$ Furthermore, the use of graphene to support CdS quantum dots and single layer $\mathrm{MoS}_{2}\left(\mathrm{~s}-\mathrm{MoS}_{2}\right)$ appears to give a higher hydrogen evolution rate. ${ }^{7}$ The functional roles of each component in these composite mixtures and their synergistic effect(s) for photocatalytic $\mathrm{H}_{2}$ evolution are not yet clear. It is believed that by elucidating such material interactions, a rational design of composite materials for more efficient photocatalytic conversion into chemicals could be developed.

Here we report the systematic study of correlation of the photocatalytic $\mathrm{H}_{2}$ activity with the structural and electronic properties of CdS and $\mathrm{s}-\mathrm{MoS}_{2}$ and interactions with surface functionalized single, double and multi-walled carbon nanotubes (SWNTs, DWNTs and MWNTs) (see the ESI $\dagger$ ) in composites using a range of characterization techniques including XRD, TEM, SAED and Raman. In particular, the time-resolved photoluminescence (TRPL) technique is invoked to investigate the lifetime of excitons with reference to activity measured for this type of composite. It is found that the exciton recombination that readily takes place on CdS upon light activation can be effectively quenched when it is mixed with carbon nanotubes (CNTs) due to rapid transfer of the photo-excited electrons to the carbon structure before the proton reduction from water for the hydrogen production over s-MoS 2 . Thus, the light capturing sites (CdS) can be separated from hydrogen production sites $\left(\mathrm{s}-\mathrm{MoS}_{2}\right)$ via CNTs as electronic mediators. A higher hydrogen production rate is achieved over the two finely dispersed phases on the CNT than without the carbon structure due to its higher electron 
conductivity and storage capacity. ${ }^{14,15}$ It is also found that typical acid functionalization of CNTs for the immobilization of CdS and $\mathrm{MoS}_{2}$ can lead to destruction of surface graphitic conjugation, affecting the essential process of electron transfer, which will lead to poor activity. As a result, DWNTs with intact inner carbon tubules act as optimal electron mediators for photocatalytic hydrogen production.

The powder XRD pattern of synthesised CdS quantum dots according to the literature is shown in Fig. S1(a) (ESI $\dagger$ ), which confirms the CdS cubic structure. The Scherrer equation revealed an average particle size of $4.76 \mathrm{~nm}$. This suggests small but uniform CdS nanoparticle size. The UV-visible absorption of CdS presented in Fig. S1(b) (ESI $\dagger$ ) features an absorption hump at $472 \mathrm{~nm}$ corresponding to a reported band gap of $2.63 \mathrm{eV} .^{3,4}$ The TEM image also reveals typical $4.32 \mathrm{~nm}$ CdS nanoparticles and their size distribution is shown in Fig. S1(c) and (d) (ESI $\dagger)$. Single layer $\mathrm{MoS}_{2}$ has also been synthesised (see the ESI $\dagger$ ). Fig. S2 (ESI $\dagger$ ) clearly shows that CdS nanoparticles tend to aggregate on the basal and edge regions of the restacked s-MoS $\mathrm{S}_{2}$ sheets with more than one monolayer thickness. The severe aggregation of CdS and $\mathrm{s}-\mathrm{MoS}_{2}$ at the material interface indicates a poor dispersion of these components for light capture and charge (exciton) separation. It was envisaged that dispersion of CdS and $\mathrm{s}-\mathrm{MoS}_{2}$ on high surface area CNTs may overcome the problems. Typical $\mathrm{HNO}_{3}$ acid pre-treatment on CNTs has been applied for the removal of contaminants (such as amorphous carbon and catalyst particles) and also functionalization of the outer carbon structure with terminal carboxyl and hydroxyl groups for the immobilization and dispersion of a solid phase in the composite. ${ }^{16}$ A detailed selection of acid treatments was reviewed, which revealed that most of the treatments can not only effectively remove contaminants but also cause shortening of tube length and functionalize the carbon surface. ${ }^{17}$ Among various acids and concentrations applied, $3 \mathrm{M} \mathrm{HNO}_{3}$ was regarded as the optimum concentration and served as a compromise between the yield of the functional groups created on CNTs and the chemical damage to the CNT structure. Therefore, the $3 \mathrm{M} \mathrm{HNO}_{3}$ treatment was chosen for the CNT pre-treatment. The photocatalytic hydrogen production activity of the composite samples with lactic acid as the sacrificial reagent was measured. Fig. 1(a) shows that CdS or CdS/DWNTs are totally inert for the hydrogen production, implying that CdS and the CdS/DWNT interface cannot provide sites for proton reduction despite the well-known light capture ability for exciton production over the CdS and DWNT phases. In contrast, when $\mathrm{s}-\mathrm{MoS}_{2}$ is used, a significant quantity of $\mathrm{H}_{2}$ is produced $\left(137 \mu \mathrm{mol} \mathrm{h}{ }^{-1} \mathrm{~g}^{-1}\right)$. Furthermore, the $\mathrm{CdS} / \mathrm{s}-\mathrm{MoS}_{2}$ composite produces $1380 \mu \mathrm{mol} \mathrm{h} \mathrm{h}^{-1} \mathrm{~g}^{-1} \mathrm{H}_{2}$, which is clearly greater than CdS or s-MoS alone. $^{7}$ There is a further increase in the amount of evolved $\mathrm{H}_{2}$ gas when DWNTs are added to the $\mathrm{CdS} / \mathrm{s}-\mathrm{MoS}_{2}$ (see E, F, G).

With an increased amount of DWNTs added, a maximum of $\mathrm{H}_{2}$ gas is evolved $\left(5728 \mu \mathrm{mol} \mathrm{h} \mathrm{h}^{-1} \mathrm{~g}^{-1}\right)$ at a $5 \mathrm{mg}$ DWNT content. However, further increase in the amount of DWNTs could impair the photocatalytic $\mathrm{H}_{2}$ production, presumably due to masking of active sites, i.e. the shielding effect, ${ }^{4}$ when $7 \mathrm{mg}$ of DWNTs is used. Upon comparing different forms of CNTs
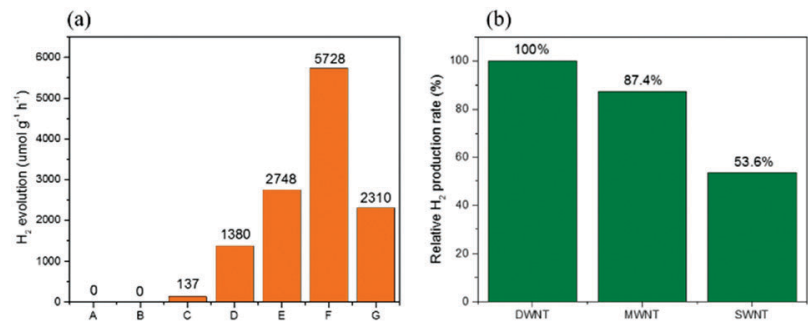

Fig. 1 (a) Hydrogen evolution rate for $20 \mathrm{mg}$ of catalysts extracted from (A) CdS, (B) CdS/DWNT, (C) s-MoS 2 , (D) CdS/s-MoS 2 , (E) CdS/2 mg DWNT/ $\mathrm{s}-\mathrm{MoS}_{2}$, (F) CdS/5 mg DWNT/s-MoS , and (G) CdS/7 mg DWNT/s-MoS 2 . (b) Hydrogen evolution rate for $5 \mathrm{mg}$ of DWNTs; MWNTs and SWNTs to support CdS and s-MoS 2 .

(SWNTs, DWNTs and MWNTs) with the same loading, see Fig. 1(b), the DWNT composite gives the best activity on a per gram basis. Noticeably, the SWNT composite gives only half a $\mathrm{H}_{2}$ production rate compared to the DWNT. As a result, the functional roles for each component particularly the nature and quality of CNTs as supports with respect to hydrogen production activity were investigated through careful material characterisation studies. To explore the charge (exciton) dynamics within the synthesised composites, the samples were examined using static and time-resolved photoluminescence spectroscopy (PL and TRPL). The steady-state PL spectra at an excitation wavelength of $405 \mathrm{~nm}$ shown in Fig. 2a contain anticipated strong emission from CdS quantum dots centred at $750 \mathrm{~nm}$ by recombination of excitons from the trap-state. However, CdS quantum dots when mixed with either CNTs or s-MoS 2 or $\mathrm{CNT} / \mathrm{s}-\mathrm{MoS}_{2}$ exhibit much smaller trap-state peaks compared with $\mathrm{CdS}$ alone, suggesting that the radiative recombination of excitons in CdS is minimised. CNTs appear to be more effective in quenching the PL than $\mathrm{MoS}_{2}$ on the same weight basis but the CdS/DWNT/s-MoS mixture is the most effective. Fig. $2 b$ shows the effectiveness
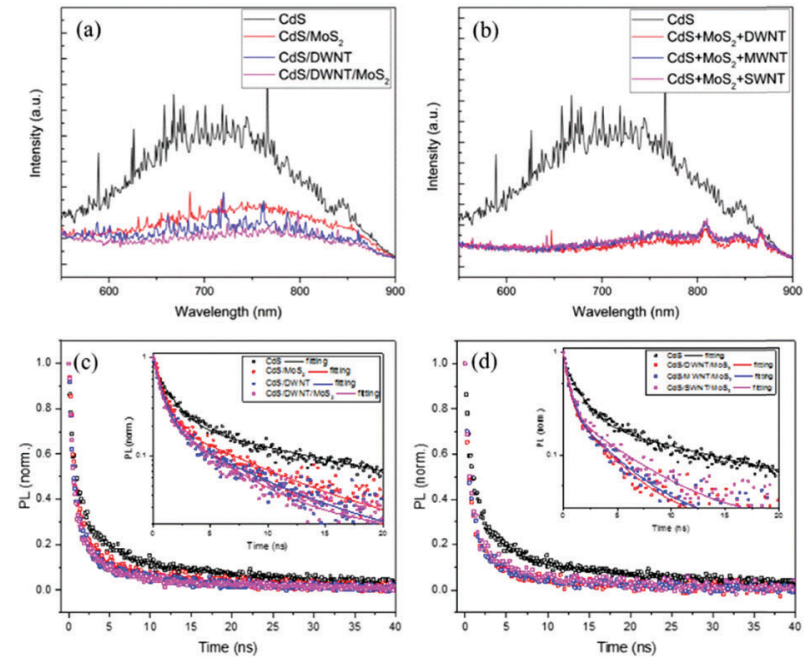

Fig. 2 Steady state PL, excitation $405 \mathrm{~nm}$, for (a) CdS samples with different components and (b) CdS and CdS/CNT/s-MoS samples. Time-resolved PL with decay monitored at $750 \mathrm{~nm}$, excitation $405 \mathrm{~nm}$, for (c) CdS samples with different components and (d) $\mathrm{CdS}$ and $\mathrm{CdS} / \mathrm{CNT} / \mathrm{s}-\mathrm{MoS}_{2}$ with different natures of CNTs. 
Table 1 The fractional contribution $\left(f_{\mathrm{i}}\right)$ and lifetime $\left(\tau_{\mathrm{i}}\right)$ of each decay component $\&$ average lifetime $\left(\tau_{\text {avg }}\right)$ for various samples

\begin{tabular}{lcllrc}
\hline Material & $f_{1}(\%)$ & $\tau_{\mathbf{1}}(\mathrm{ns})$ & $f_{2}(\%)$ & $\tau_{\mathbf{2}}(\mathrm{ns})$ & $\tau_{\text {avg }}(\mathrm{ns})$ \\
\hline $\mathrm{CdS}$ & 20.8 & 1.45 & 79.2 & 16.03 & 13.00 \\
$\mathrm{CdS} / \mathrm{MoS}_{2}$ & 27.3 & 0.74 & 72.7 & 7.82 & 5.88 \\
CdS/DWNT & 30.9 & 0.79 & 69.1 & 6.83 & 4.97 \\
CdS/DWNT/MoS & 28.4 & 0.64 & 71.6 & 3.96 & 3.02 \\
CdS/MWNT/MoS & 33.9 & 0.89 & 66.1 & 4.72 & 3.42 \\
CdS/SWNT/MoS & 26.8 & 0.79 & 73.2 & 5.90 & 4.53
\end{tabular}

of DWNTs, MWNTs and SWNTs in quenching the trap-state emission PL. From TRPL (Fig. $2 \mathrm{c}$ and d), an average lifetime $(\tau)$ for exciton recombination for each sample was derived and is shown in Table 1. The pristine CdS demonstrates an average lifetime of $13.00 \mathrm{~ns}$; upon mixing with s-MoS ${ }_{2}$ and DWNTs, the photo-generated carriers are indeed significantly quenched, where the DWNTs again show better quenching ability compared to $\mathrm{s}-\mathrm{MoS}_{2}$. This indicates that the collection/extraction of photoexcited electrons from CdS to s-MoS 2 via DWNTs is more efficient than that of $\mathrm{CdS} / \mathrm{s}-\mathrm{MoS}_{2}$, presumably due to the electron storage and mobility of DWNTs. Among all the samples, CdS/DWNT/ s-MoS ${ }_{2}$ displays the lowest $\tau$ (3.02 ns). Similar to the static PL, SWNTs (4.53 ns) are less effective than MWNTs (3.42 ns) and DWNTs (3.02 ns) in quenching the exciton emission. $\mathrm{As} \mathrm{MoS}_{2}$ is only the component to provide active sites for proton reduction to hydrogen (see Fig. 1), it is likely that the photoelectrons generated by CdS upon light excitation are quickly taken up by the CNTs before passing to s-MoS 2 . Apparently, the nature of CNTs appears to be critical for efficient transport and storage of photoelectrons from the CdS phase.

It is well known that the electronic properties of carbon nanotubes are crucially dependent on the diameter and chirality due to their distorted conjugated atomic tubular structure with partial overlap of Pz orbitals, whereas the electronic properties of MWNTs are quite complex, as each layer in the MWNTs can have different chiralities. Furthermore, MWNTs show considerably lower electronic conductivity than those of SWNTs or DWNTs because the interactions between the layers within MWNTs were found to disturb the electrical current along the tube axis. ${ }^{15}$

The morphology of DWNTs is very close to SWNTs, but the inner carbon tubes may provide detainment of the conjugation atomic pathway from aggressive mechanical or chemical damage to the outer tube surface. ${ }^{17}$ High-resolution TEM (HR-TEM) images (Fig. 3a and b) thus show the raw single and doublewalled CNTs with the tubular graphene feature for the SWNT and DWNT, respectively. After $3 \mathrm{M} \mathrm{HNO}_{3}$ treatment, the majority of SWNTs are converted into carbon sheets and amorphous carbon (the area of serious destruction of SWNTs to amorphous carbon is shown in Fig. 3c). But many of the DWNTs retain their partially destructed tubular structure with amorphous carbon fragments (Fig. 3d). Thus, the more fragile nature of SWNTs leading to opening, unfolding and destruction to carbon fragments is particularly noted. Fig. S3 (a) (ESI $\dagger$ ) shows the formation of new peaks of $\mathrm{C}=\mathrm{O}$ stretching at $1691 \mathrm{~cm}^{-1}, \mathrm{C}=\mathrm{C}$ and $\mathrm{C}=\mathrm{O}$ at $1571 \mathrm{~cm}^{-1}$ and $\mathrm{C}-\mathrm{O}$ stretching at $1064 \mathrm{~cm}^{-1}$ for the acid treated CNTs by FTIR spectroscopy. To quantify the global structural

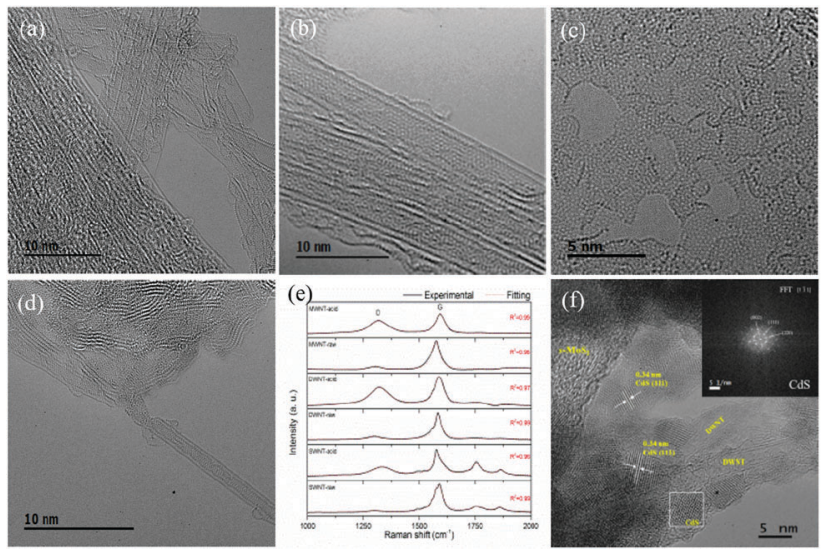

Fig. 3 HR-TEM images of (a) raw SWNTs, (b) raw DWNTs, (c) acid-treated SWNTs, and (d) acid-treated DWNTs. (e) Raman spectra of raw CNTs and 3 M $\mathrm{HNO}_{3}$ treated CNTs. (f) CdS/DWNT/s-MoS 2 (sheets) with inset of fast-Fourier transform (FFT) of CdS. The enlarged images are found in Fig. S2 (ESI $\dagger$ ).

change of the samples before and after the same acid treatment, Raman spectra of raw and $\mathrm{HNO}_{3}$-treated CNTs are shown in Fig. 3e. The band located around $1315 \mathrm{~cm}^{-1}$ is assigned to the D-band which is commonly associated with the disordered, $\mathrm{sp}^{3}$-hybridised carbon arising from defects and impurities, while the band located around $1580 \mathrm{~cm}^{-1}$ is assigned to the G-band which is associated with the crystalline graphitic structures. ${ }^{18}$ The intensity ratio of the D-band to the G-band is commonly used to quantify the degree of disorder in a graphene structure. ${ }^{19}$ Generally, $\mathrm{HNO}_{3}$-treated CNTs showed larger D-band to G-band ratios, indicating the destruction of the graphene structure of CNTs during the acid treatment. Notably, the $I_{\mathrm{D}} / I_{\mathrm{G}}$ ratio increases to 5.2 times in the SWNT, followed by 4.9 times in the DWNT and 2.1 times in the MWNT sample. The increase of the D-band ratio in SWNTs suggests that SWNTs indeed have undergone more serious damage after identical acid treatment (particularly on surfaces), which we believe impairs their charge transfer ability (larger $\tau$ shown in Table 1), hence resulting in lower $\mathrm{H}_{2}$ activity compared to DWNTs (see Fig. 1b). Fig. 3f shows that the image of the CdS/DWNT/s-MoS ${ }_{2}$ composite, in which the regions of CdS nanoparticles and s-MoS 2 sheets dispersed on the partial tubular carbon structure can be differentiated through the corresponding lattice fringe distances and fast-Fourier transform (FFT) (inset of Fig. 3f). The intimate contact between $\mathrm{CdS}, \mathrm{s}-\mathrm{MoS}_{2}$ and carbon nanotubes are clearly evidenced, which suggests that the CNTs remarkably decrease the aggregation of CdS and s-MoS 2 as they spread uniformly in the nanocomposite for superior $\mathrm{H}_{2}$ activity compared to that without the carbon support. This indicates the future design of efficient photoactive composites for separation and optimization of light capture nanomaterials from catalytic hydrogen production materials via electron conductive mediators in a remote way. It is also clear from this study that the crucial maintenance of the integrity of the graphitic tubular structure and the intactness of the conjugation of inner tubes are imperatively important for the charge transport and storage from the light capture CdS phase to hydrogen production sites on $\mathrm{s}-\mathrm{MoS}_{2}$ for optimal photo-catalysis. 


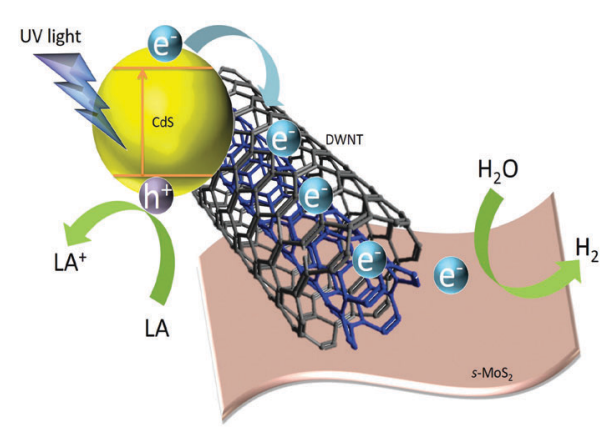

Scheme 1 Photocatalytic production of hydrogen from water over CdScarbon nanotube (CNT)-MoS 2 is found critically dependent on the content and nature of the nanotube used. Time resolved photo-luminescence indicates that the surface graphitic structure of the CNT is vulnerable to damage, halting the essential electron transfer for $\mathrm{H}_{2}$ formation. Thus, the DWNT exhibits superior $\mathrm{H}_{2}$ activity to the SWNT because of the structural integrity of the inner tubule.

In summary, photocatalytic hydrogen evolution activity through water splitting is greatly enhanced by the incorporation of carbon nanotubes and single-layered $\mathrm{MoS}_{2}$ nano-sheets as cocatalysts into the CdS system. CdS/DWNT/s-MoS 2 with the optimal amount of DWNTs (mass ratio of CdS : DWNT: $\mathrm{s}-\mathrm{MoS}_{2}=100: 5: 2$ ) can provide a high hydrogen evolution rate of $5728 \mu \mathrm{mol} \mathrm{g} \mathrm{g}^{-1} \mathrm{~h}^{-1}$, and the unique roles of intact carbon nanotubes as electron mediators are for the first time identified by this present systematic TRPL study, as summarized in Scheme 1. In addition, a DWNT shows the best performance compared to other forms of CNTs, due to its robust double-walled structure, which can provide protection for the inner tube from surface damage during functionalization/handling, while a SWNT inevitably suffers from destruction of conjugation. This study depicts the importance of fabrication of intimate heterojunctions demonstrating the benefits of nano-ensembles of functional units for light capture, carrier transfer and catalysis in synergy for efficient photo-production of hydrogen from water.

The financial supports of this work from the EPSRC of UK and the Royal Society international joint project with the carbon nanotube group (CNRS) at Toulouse, France, are acknowledged. MMJL acknowledges a Swire Scholarship for her DPhil study at Oxford University, U.K.

\section{Notes and references}

$1 \mathrm{M}$. Ni, M. K. Leung, D. Y. Leung and K. Sumathy, Renewable Sustainable Energy Rev., 2007, 11, 401-425.

2 A. Kudo and Y. Miseki, Chem. Soc. Rev., 2009, 38, 253-278.

3 X. Chen, S. Shen, L. Guo and S. S. Mao, Chem. Rev., 2010, 110, 6503-6570.

4 Q. Li, B. Guo, J. Yu, J. Ran, B. Zhang, H. Yan and J. Gong, J. Am. Chem. Soc., 2011, 133, 10878-10884.

5 A. Cao, Z. Liu, S. Chu, M. Wu, Z. Ye, Z. Cai, Y. Chang, S. Wang, Q. Gong and Y. Liu, Adv. Mater., 2010, 22, 103-106.

6 D. J. Fermin, E. A. Ponomarev and L. M Peter, J. Electroanal. Chem., 1999, 473, 192-203.

7 T. Jia, A. Kolpin, C. Ma, R. C.-T. Chan, W.-M. Kwok and S. C. E. Tsang, Chem. Commun., 2014, 50, 1185-1188.

8 J. Zhang, Z. Zhu and X. Feng, Chem. - Eur. J., 2014, 20, 10632-10635.

9 I. Robel, B. A. Bunker and P. V. Kamat, Adv. Mater., 2005, 17, 2458-2463.

10 D. Lang, T. Shen and Q. Xiang, ChemCatChem, 2015, 7, 943-951.

11 S. Pan and X. Liu, New J. Chem., 2012, 36, 1781-1787.

12 Q. Xiang, F. Cheng and D. Lang, ChemSusChem, 2016, 9, 996-1002.

13 A. Ye, W. Fan, Q. Zhang, W. Deng and Y. Wang, Catal. Sci. Technol., 2012, 2, 969-978.

14 R. Baughman, A. Zakhidov and W. A. de Heer, Science, 2002, 297, 787-792.

15 R. Saito, G. Dresselhaus and M. S. Dresselhaus, Phys. Rev. B: Condens. Matter Mater. Phys., 1996, 53, 2044-2050.

16 Y. Yao, G. Li, S. Ciston, R. M. Lueptow and K. A. Gray, Environ. Sci. Technol., 2008, 42, 4952-4957.

17 T. Bortolamiol, P. Lukanov, A.-M. Galibert, B. Soula, P. Lonchambon, L. Datas and E. Flahaut, Carbon, 2014, 78, 79-90.

18 I. D. Rosca, F. Watari, M. Uo and T. Akasaka, Carbon, 2005, 43, 3124-3131.

19 H. Murphy, P. Papakonstantinou and T. I. T Okpalugo, J. Vac. Sci. Technol., B: Microelectron. Nanometer Struct.-Process., Meas., Phenom., 2006, 24, 715-720. 